\title{
Effect of disturbance on parameters derived from self-boring pressuremeter tests in sand
}

\author{
M. FAHEY and M. F. RANDOLPH (1984). Géotechnique 34, No. 1, 81-97
}

Dr B. G. Clarke, PM Insitu Techniques Ltd, and Professor C. P. Wroth, Department of Engineering Science, University of Oxford

The Paper, an important contribution to the use of the self-boring pressuremeter (SBP), presents the results of field trials into the drilling of the SBP into sand. It highlights the difficulties of drilling into sand and shows, using the drilling techniques described, that there is considerable disturbance to the surrounding soil. Because of this disturbance the analysis of Hughes, Wroth \& Windle (1977) can no longer be applied. In this Discussion an empirical method is presented to make allowances for the disturbance. It would be preferable to improve the drilling technique and to minimize the disturbance further. We have carried out numerous tests in a variety of sand deposits and we discuss our conclusions below.

There are several aspects of the drilling technique which can cause disturbance. These are briefly mentioned in this Discussion and are discussed in detail by Clarke (1981). To summarize they are

(a) possible vibrations transmitted from the drilling equipment at the surface

(b) pressure and flow rate of the drilling fluid

(c) position of the cutter

(d) rate of advance of the instrument and speed of rotation of the cutter

(e) shear at the instrument-soil interface.

The design of the equipment minimizes items (a) and (e), and the operator can control items (b)-(d). The Authors quite correctly state that one of the worst problems in drilling in sand with water is the effect of scouring which can result in the instrument being wash bored into the ground. The solution put forward is to use an auger-type cutter so reducing the scouring action. However, this still results in some disturbance and therefore a semi-empirical approach to the analysis of the tests is proposed.

One of the most important aspects of SBP drilling is to balance the in situ stresses at the leading edge of the instrument. This is partly achieved by adjusting the cutter position so that the force required to extrude the soil into the shoe balances the overburden pressure. To do this requires a conical shoe and ideally the cutter described by Windle (1976). This technique has heen successfully used in clays (Clarke, 1981; Wroth, 1982). Both Windle (1976) and Fahey (1980) found that this cutter, when used in sands, causes scouring and as a result the augertype cutter was proposed. Unfortunately, although this reduces the action of scouring, the principle of self-boring no longer applies as the extrusion of sand into the parallel-sided shoe does not create sufficient pressure to balance the overburden stress. This means there is still some disturbance due to stress relief at the leading edge of the SBP. So the empirical analysis still has to be used. Furthermore, we have found the auger-type cutter can only be used in loosemedium fine sand.

We have found by using drilling mud and carefully controlling items $(b)$ and $(d)$, though still using the spade-type cutter and conical shoe, that we were able to drill the SBP into sand and to carry out tests which show very little disturbance if any at all (Fig. 1). The use of the spade-type cutter and conical shoe means that the principle of self-boring is maintained and the use of drilling mud prevents the action of scouring. Thus the need to estimate the strain at which the applied pressure equals the lateral stress no longer applies as we are able to estimate the lateral stress directly from the test curve and this occurs at very nearly zero strain. It should be stressed that for tests in sand and in clay the pressure at which the displacement transducers show the membrane beginning to move is not necessarily the lateral stress: account has to be taken of the membraneinstrument behaviour.

The sccond aspect of the Paper discusses the analysis of the test. If there is very little disturbance to the soil then the analysis of Hughes $e t$ al. (1977) with no zero adjustment applies; this is what we use as the disturbance is kept to a minimum because of the improved drilling technique. We have made use of the zero adjustment technique presented in the Paper, after discussions with Fahey (1979), for those tests in 
which there may have been some disturbance. We have found that the rules applied do work but it must be emphasized that the membrane must be expanded by a sufficient amount so that the sand beyond the zone disturbed by the drilling is taken to the limit stress ratio. The amount

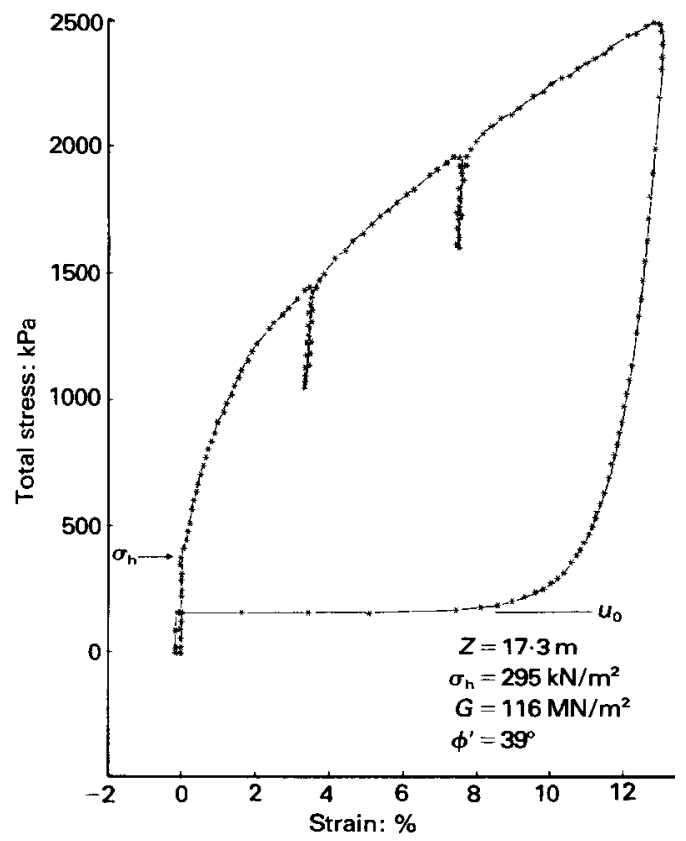

Fig. 1. Typical drained test in sand

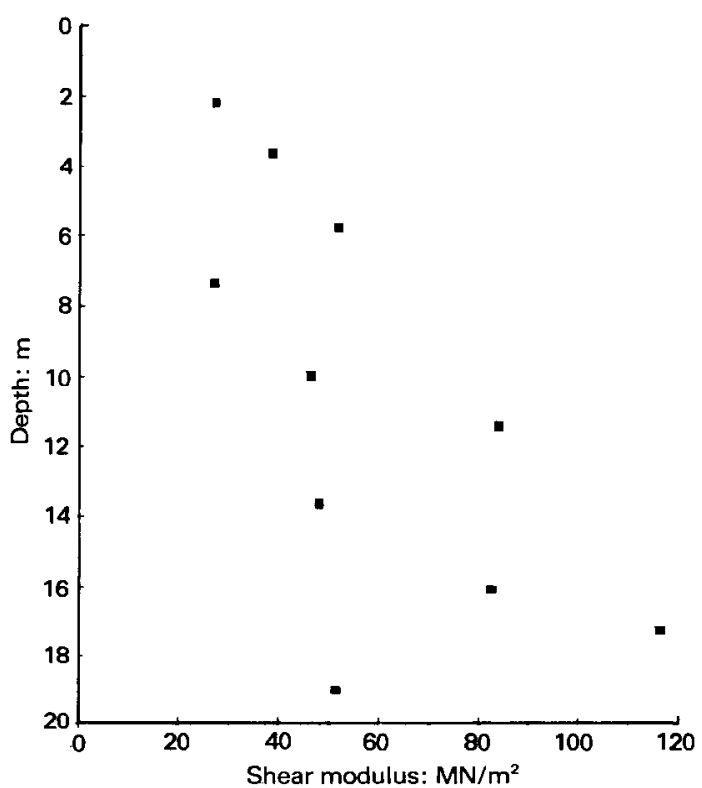

Fig. 2. Variation in shear modulus with depth of disturbance indicated in the Paper for a flush shoe is up to about $1 \%$ cavity strain, though we have found that the disturbance using the drilling techniques described in the Paper can be greater. Our instrument is capable of being expanded up to $20 \%$ cavity strain thus ensuring that undisturbed soil is tested.

Both in clays and in sands the initial tangential shear modulus immediately after the lateral stress is reached is suspect especially if there is any disturbance. A more reliable value can be found from the unload-reload cycle. The point at which the cycle is carried out and the magnitude of the cycle are important. Again the cycle should be carried out at sufficient cavity strain to ensure that undisturbed material is being tested and the magnitude of the cycle should be such that the soil does not fail in extension. This value of modulus when plotted against depth does show some scatter. However, the modulus of sand varies according to the mean effective stress at which the modulus loop was carricd out. Tests were carricd out in a medium dense sand of uniform grading using the drilling techniques described here. In Fig. 2 the modulus values are plotted against depth and show much scatter whereas if the modulus values are plotted against the mean effective stress the amount of scatter is reduced as shown in Fig. 3.

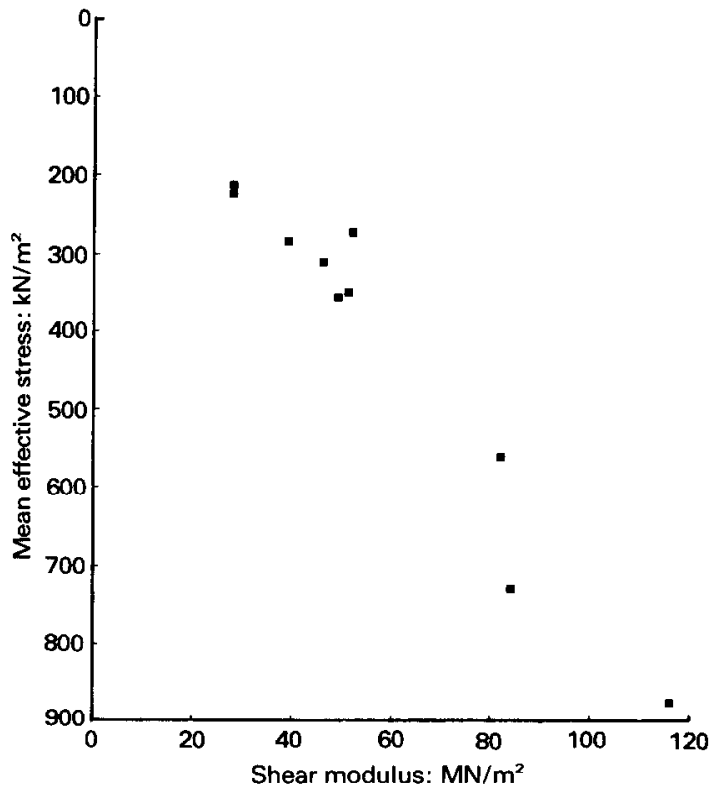

Fig. 3. Variation in shear modulus with mean effective stres: 
So by improved drilling techniques it is possible to obtain valid values of lateral stress in sand. If this is the case then the empirical analysis postulated in the Paper does not apply and in situ values of the angle of friction can be found directly from the field stress-strain curve. Finally, shear modulus is best measured over an unload-reload cycle and should be quoted together with the mean effective stress at which the modulus was measured.

\section{REFERENCES}

Clarke, B. G. (1981). In situ testing of clays using the Cambridge self-boring pressuremeter. $\mathrm{PhD}$ thesis, Cambridge University.

Fahey, M. (1979). Private communication.

Fahey, M. (1980). A study of the pressuremeter test in dense sand. $\mathrm{PhD}$ thesis, Cambridge University.

Hughes, J. M. O., Wroth, C. P. \& Windle, D. (1977). Pressuremeter tests in sands. Géotechnique 27, No. 4, 455-477.

Windle, D. (1976). In situ testing of soils with a selfboring pressuremeter. $\mathrm{PhD}$ thesis, Cambridge University.

Wroth, C. P. (1982). British experience with the selfboring pressuremeter. Symp. Pressuremeter and its Marine Applications, Paris, April 1982.

\section{Authors' reply}

The Authors fully support the Writers' statement that, instead of trying to make allowance for the effects of disturbance, 'it would be preferable to improve the drilling technique and to minimize the disturbance further'. This statement echoes the major conclusion stated in our Paper, that 'while it may be possible to correct the values of $\phi^{\prime}$ even when considerable disturbance has been caused during drilling, it is preferable to take as much care as possible during drilling so that there is no need to resort to correction procedures'. We aimed to show in our Paper that, using what were state of the art drilling procedures at that time (early 1980), credible values of strength and stiffness parameters for sand could be obtained, provided that reasonable care was exercised. If some disturbance did occur, this could be recognized from the appearance of the test curve, especially when plotted as effective cavity pressure versus cavity strain on logarithmic axes. That progress has since then been made in improving installation procedures, as outlined by the Writers, is only to be welcomed.

The Writers correctly point out that the shear modulus of sand could be expected to correlate better with the mean effective stress in the sand at the time of performing an unload-reload loop than with depth. However, a major problem in attempting such a correlation is to determine the appropriate value of mean effective stress in the soil at the time the loop is begun. If it is accepted that sand can be modelled as an elastic, frictional plastic material, with dilation (or contraction) only in the plastic state, then it can be shown that the mean effective stress does not change around the pressuremeter in the elastic zone, but that in the plastic zone the mean stress does increase. Further, accepting the soil model on which the analysis of Hughes et al. is based, it can be shown that the value of the plane strain mean effective stress parameter $s^{\prime}=\left(\sigma_{r}{ }^{\prime}+\sigma_{\theta}{ }^{\prime}\right) / 2$ in the plastic zone decreases with radius from the centre of the instrument, becoming equal to the in situ horizontal stress at the elastic-plastic interface, i.e.

$$
\begin{aligned}
& \Delta S_{\mathrm{p}}{ }^{\prime} \propto\left(\frac{1}{r}\right)^{1-N} \\
& S_{\mathrm{p}}{ }^{\prime}=\Delta{S_{\mathrm{p}}}^{\prime}+\sigma_{\mathrm{h}}{ }^{\prime}
\end{aligned}
$$

where $N=\left(1-\sin \phi^{\prime}\right) /\left(1+\sin \phi^{\prime}\right)$ and the subscript $p$ denotes the plastic zone. If the vertical effective stress in the plastic zone is assumed to be equal to the average of the radial and hoop stresses at every radius, then the distribution of mean effective stress $p^{\prime}$ with distance from the pressuremeter can be determined.

During an unload-reload loop, all the material is inside the Mohr-Coulomb failure surface, so that assuming elastic behaviour the mean effective stress does not change during the loop. Thus, if the shear modulus is a function of mean effective stress, the gradient of the unloadreload loop is a measure of some average value of shear modulus which is higher than the in situ modulus, but lower than the modulus of the soil adjacent to the pressuremeter. In effect the instrument at this stage of the test is embedded in an elastic material with stiffness decreasing with radius, and this would invalidate (though perhaps only to a minor extent) the simple elastic analysis which leads to a unique value of the shear modulus.

It may be acceptable, for the proposed correlation exercise, to assume that the unload-reload response is dominated by the soil immediately around the pressuremeter, and therefore the value of shear modulus obtained can be correlated with the maximum mearl effective stress, i.e. the mean effective stress at the soilinstrument interface. This then implies that if any disturbance is caused during drilling, the measured value of shear modulus will be dominated by the stiffness of the disturbed zone of sand around the pressuremeter and the value 
obtained will relate more to the disturbed material than to the in situ material. This does not agree with one of our conclusions that the 'values of $G_{\mathrm{ur}} \ldots$ seem to be [the parameter] least affected by drilling disturbance'.

The Writers also suggest that drilling procedures have been perfected to such an extent that disturbance can be eliminated almost completely, and in particular that the true in situ horizontal total stress can be deduced from the 'lift-off' pressure. This implies that the stress in the soil, even immediately adjacent to the instrument, has not been affected by the insertion process. Consider what would occur if a frictionless, infinitely thin hollow cylinder is pushed into a sand deposit. Because of the interlocking or packing of the sand grains, some movement of grains would have to occur to allow passage of the cylinder. This movement would be at least of the order of half the grain diameter. In the test reported by the Writers (their Fig. 1), a radially inward movement equivalent to a cavity strain of about $0 \cdot 13 \%$ is all that would be required to reduce the in situ horizontal stress at the instrument-soil interface to zero, if the quoted horizontal stress and shear modulus values are adopted, i.e.

$$
\text { strain }=\frac{\sigma_{\mathbf{h}}}{2 G}=\frac{295}{2 \times 116000}=0 \cdot 13 \%
$$

This is equivalent to a radial movement of only $0.05 \mathrm{~mm}$. Thus, even if the in situ stresses can be balanced in the ground ahead of the cutting edge, it is difficult to accept that the cutting edge itself will not cause movements greater than this value, given that the grain size for sand may be as large as $2 \mathrm{~mm}$ (British Standard definition). Such movements might of course be radially outwards, which would cause an increase, rather than a decrease, in stress on the instrument-soil interface.

Figure 5(b) in our Paper shows that in our instrument there are some surface irregularities at the point of transition between the drilling shoe and the body of the instrument. These irregularities alone could be sufficient to affect significantly the stress on the soil-instrument interface, even if the horizontal stress had not already been affected by the leading edge of the drilling shoe.

Therefore, given the high stiffness values measured in all but the loosest sands, we feel that it is very optimistic to expect that any insertion technique, no matter how perfect, will not affect the horizontal stress acting on the instrument. We showed in our Paper (refer to Fig. 20) that, once this stress was altered, there was very little reason to expect that the test curve would exhibit any change in behaviour when the cavity pressure reached the value of the in situ horizontal stress.

Finally, with regard to the choice between the various shear modulus values which can be measured in the test (initial, unload-reload and final unload values), we agree with the Writers that the unload-reload modulus $G_{u r}$ can be more reliably measurcd than the initial modulus $G_{i}$. The first Author has performed many selfboring pressuremeter tests in dune and alluvial sands in the Perth area of Western Australia. In most cases, the question arises whether the initial or unload-reload modulus values are the most appropriate for use in settlement calculations. Except where high stress ratios (i.e. high shear strains) are expected, the usual recommendation has been to use the unload-reload values to give the best prediction of settlement, but to use the initial values to give a more conservative upper-bound estimate. However, there is very little definite evidence available on which to base such a recommendation. Therefore, although the value of $G_{u r}$ can be measured with greater confidence, the more important question whether it is the most appropriate value for settlement prediction in sand still remains. It is only by careful monitoring of structure performance, and comparison with predictions based on pressuremeter-derived parameters, that this question will be resolved. 